Proyecciones

Vol. 27, No 1, pp. 15-28, May 2008.

Universidad Católica del Norte

Antofagasta - Chile

\title{
ECUACIÓN DE ENSKOG CON TÉRMINO FUERZA *
}

\author{
RAFAEL GALEANO ANDRADES \\ BERNARDO OROZCO HERRERA \\ and \\ MARÍA OFELIA VÁSQUEZ ÁVILA \\ UNIVERSIDAD DE CARTAGENA, COLOMBIA
}

Received: November 200\%. Accepted : January 2008

\begin{abstract}
La meta en este paper es probar existencia y unicidad de soluciones para la ecuación de Enskog con término fuerza

The goal in this paper is prove existence and uniqueness of solutions for the Enskog equation with force term.
\end{abstract}

Keywords : Ecuación de Enskog, término fuerza.

Subjclass : 35Q75,82-02.

*Este artículo es un resultado del proyecto de investigación "Ecuaciones Elipticas de Tipo Cinético", financiado por la Universidad de Cartagena. 


\section{INTRODUCCIÓN}

La Ecuación de Enskog es una modificación de la Ecuación Cinética de Boltzmann, en la cual cada partícula es considerada como una esfera dura con diámetro no cero $a>0$ (y además las colisiones toman lugar en un punto a la distancia $\frac{a}{2}$ de los centros de las partículas de colisión), la ecuación de Enskog da una buena descripción del fenómeno del transporte en gases densamente moderados y es escrita como:

$$
\left\{\begin{array}{l}
\frac{\partial f}{\partial t}+v \cdot \nabla_{x} f+F \cdot \nabla_{v} f+t \frac{\partial F}{\partial t} \cdot \nabla_{v} f=E(f) \\
f(0, x, v)=f_{0}(x, v)
\end{array}\right.
$$

donde $(x, v) \in \mathbf{R}^{3} \times \mathbf{R}^{3}$ y $E(f)$ es el operador de Enskog abstracto dado por $E(f)=E^{+}(f)-E^{-}(f)$ con

$$
\begin{aligned}
& \mathrm{E}^{+}(f)=a^{2} \int_{\mathbf{R}^{3} \times S_{+}^{2}} Y(f) B(\eta, w-v) f\left(t, x, v^{\prime}\right) f\left(t, x+a \eta, w^{\prime}\right) d w d \eta \\
& \mathrm{E}^{-}(f)=a^{2} f(t, x, v) \int_{\mathbf{R}^{3} \times S_{+}^{2}} Y(f) B(\eta, w-v) f(t, x-a \eta, w) d w d \eta
\end{aligned}
$$

donde $S_{+}^{2}=\left\{\eta \in \mathbf{R}^{3}:|\eta|=1, B(\eta, w-v) \geq 0\right\} \mathrm{y}$

$$
\left\{\begin{array}{c}
v^{\prime}=v+\eta B(\eta, w-v) \\
w^{\prime}=w-\eta B(\eta, w-v)
\end{array}\right.
$$

con $B \in L_{l o c}^{1}\left(S_{+}^{2} \times \mathbf{R}^{3}\right)$ un operador continuo y acotado.

El término fuerza $F$,

$$
F:(0, \infty) \times \mathbf{R}^{3} \times \mathbf{R}^{3} \underset{(t, x, v)}{\longrightarrow \mathbf{R}^{3}} \longmapsto F(t, x, v)=\frac{1}{t} x-v+\left(\frac{1}{t}, \frac{1}{t}, \frac{1}{t}\right)
$$

Tiene las siguientes propiedades

$$
\frac{\partial F_{i}}{\partial x_{j}}=\{1 t, \text { si } i=j, 0, \text { si } i \neq j
$$

$\mathrm{y}$

$$
\frac{\partial F_{i}}{\partial v_{j}}=\{-1, \text { si } i=j, 0, \text { si } i \neq j .
$$

La definicion de F implica que:

$$
-v-F-t \frac{\partial F}{\partial t}=0
$$

Estos campos son en general de la forma: $F(t, x, v)=\frac{1}{t} x-v+g\left(\frac{1}{t}\right)$, siendo $g\left(\frac{1}{t}\right) \in R^{3}$ no lineal y se usan para 
representar lo que los físicos llaman SINGULARIDAD ORIGINAL [SO] en los modelos del Bing-Bang, ver [1].

Sea

$$
V=\left\{f \in L_{l o c}^{1}\left([0, T] \times \mathbf{R}^{3} \times \mathbf{R}^{3}\right): 0 \leq f(t, x, v) \leq \hat{f}(t, x, v)\right\}
$$

con

$$
\|f\|_{V}=\sup _{t \in[0, T], x \in \mathbf{R}^{3}, v \in \mathbf{R}^{3}}\left|\frac{f(t, x, v)}{\hat{f}(t, x, v)}\right|
$$

donde $\widehat{f}(t, x, v)=c(t) \exp \left[-\frac{1}{2}\left|\frac{x-v t-F t}{b}\right|^{2}\right]$ y $b>0, \quad c: \mathbf{R} \rightarrow \mathbf{R}^{+}$. El espacio $V$ es de Banach con la norma definida anteriormente(ver MISCHLER S. and PERTHAME B.(2000)[11], pag. 1022).

Al funcional $Y(f)$ definido sobre el espacio $V$ imponemos las siguientes hipótesis:

1. Existe una constante $M>0$ tal que $|Y(f)| \leq M$, es decir, es uniformemente acotado.

2. $a \geq 0$ y $0 \leq f_{1} \leq f_{2} \Rightarrow Y\left(f_{1}\right) \leq Y\left(f_{2}\right) . \quad$ (monotonia)

3. $Y$ es Lipschitz continuo, esto es existe un $K \geq 0$ tal que $\mid Y\left(f_{1}\right)-$ $Y\left(f_{2}\right)|\leq K| \mid f_{1}-f_{2} \|_{V}$, Como una consecuencia de (2) se tiene que $E\left(f_{1}\right) \leq E\left(f_{2}\right)$ si $f_{1} \leq f_{2}$.

La literatura matemática sobre el análisis cualitativo del problema de Cauchy para la ecuación de Boltzmann, en la presencia de campo externo, es poca comparable con la literatura del problema de Cauchy para la ecuación sin campo externo.

Consideremos primero la ecuación espacialmente homogénea para partículas neutrales con un campo externo $F=F(t, x, v)$, en este tema el artículo de ASANO KIYOSHI(1987) [2], necesita ser citado. Existencia local es probada con condiciones iniciales generales y la formulación de Asano es el punto de partida de todos los estudios sobre el problema.

Otros artículos relacionados con el problema son [2],[4], [7] y [8]. En particular los artículos de ASANO KIYOSHI(1987)[2] y $\operatorname{GRUNFELD(1985)[7],~}$ 
prueban existencia global para la solución con condiciones iniciales cerca al equilibrio y un campo de fuerza conservativo del sistema tendiendo al equilibrio.

HAMDACHE(1988)[9], resolvió el problema con un dato inicial decayendo exponencialmente a cero en el espacio fase y trayectorias preescritas por un campo oscilante. Más general es el resultado de BELLOMO N, LACHOWICZ M, PALCZWISKI and TOSCANI G.(1989)[4], donde existencia global es probada para dato decayendo y para un campo de fuerza general actuando en un intervalo de tiempo $[0, T]$ con $T$ grande pero finito, éste resultado fue extendido en parte por GALEANO y PREZ(2003)[7] a la ecuación de Enskog.

El resultado que presentaremos en este artículo desarrolla una técnica diferente en el sentido del tratamiento del término Fuerza y las soluciones en el espacio $L_{l o c}^{1}$, y lo organizamos de la siguiente manera: Probamos la existencia de una supersolución de la ecuación la cual sirve para demostrar que el operador definido mas adelante mapea $V$ en $V$, luego mediante aplicación del teorema del punto fijo de Banach verificamos existencia y unicidad de la solución. No conocemos resultado análogo para la ecuación de Enskog en la literatura.

Solución de la ecuación de Enskog. Decimos que $f \geq 0, f \in$ $L_{l o c}^{1}\left([0, T] \times \mathbf{R}^{3} \times \mathbf{R}^{3}\right)$ es una solución de (1.1) si para cualquier $0<T<\infty$, $E^{ \pm}(f)(\cdot, x, v) \in L_{l o c}^{1}[0, T]$, c.t.p. en $(x, v) \in \mathbf{R}^{3} \times \mathbf{R}^{3}$ y satisface:

$$
f(t, x, v)-f(s, x, v)=\int_{s}^{t} E(f)(\tau, x, v) d \tau, \quad 0 \leq s \leq t \leq T .
$$

A continuación establecemos el teorema a demostrar

Teorema 1.1. Sean $f_{0}(x, v) \in V, F$ que satiface (1.2), (1.3) y (1.4) y $Y$ que satifacen las hipótesis 1., 2. y 3. dadas anteriormente. Además $B\langle\eta, w-v\rangle \in L_{l o c}^{1}\left(S_{+}^{2} \times \mathbf{R}^{3}\right)$ para cualquier $v \in \mathbf{R}^{3}, c(0) \leq \frac{1}{4 L M a^{2}+2 a^{2} M L T}$ con $T \geq t \geq 0$. Para $G(t, x, v)=-\frac{1}{2}\left|\frac{x-v t-F t}{b}\right|^{2}$ el siguiente problema

$$
\left\{\begin{array}{l}
\frac{\partial f}{\partial t}+v \cdot \nabla_{x} f+F \cdot \nabla_{v} f+t \frac{\partial F}{\partial t} \cdot \nabla_{v} f=E(f) \\
f(0, x, v)=f_{0}(x, v)
\end{array}\right.
$$

posee una única solución en el espacio $V$. 


\section{DESARROLLO}

Sea $\widehat{f}(t, x, v)=c(t) \exp \left[-\frac{1}{2}\left|\frac{x-v t-F t}{b}\right|^{2}\right] \operatorname{con} b>0, \mathrm{y} c: \mathbf{R} \rightarrow \mathbf{R}^{+}-\{0\}$ continuamente diferenciable, a determinar, de manera que $\widehat{f}(t, x, v)$ satisfaga la ecuación (1.1). En efecto, calculamos

$$
\begin{aligned}
\frac{\partial \widehat{f}}{\partial t} & =\frac{\partial}{\partial t}\left[c(t) \exp \left[-\frac{1}{2}\left|\frac{x-v t-F t}{b}\right|^{2}\right]\right] \\
& =c^{\prime}(t) \exp (G)+c(t) \exp (G)(G)^{\prime}
\end{aligned}
$$

Aquí

$$
\begin{aligned}
G(t, x, v)=-\frac{1}{2} & {\left[\left(\frac{x_{1}-v_{1} t-F_{1} t}{b}\right)^{2}+\left(\frac{x_{2}-v_{2} t-F_{2} t}{b}\right)^{2}+\left(\frac{x_{3}-v_{3} t-F_{3} t}{b}\right)^{2}\right] } \\
G^{\prime} & =-\frac{1}{b^{2}}\left(x_{1}-v_{1} t-F_{1} t\right)\left(-v_{1}-F_{1}-t \frac{\partial F_{1}}{\partial t}\right) \\
& -\frac{1}{b^{2}}\left(x_{2}-v_{2} t-F_{2} t\right)\left(-v_{2}-F_{2}-t \frac{\partial F_{2}}{\partial t}\right) \\
& -\frac{1}{b^{2}}\left(x_{3}-v_{3} t-F_{3} t\right)\left(-v_{3}-F_{3}-t \frac{\partial F_{3}}{\partial t}\right)
\end{aligned}
$$

por lo tanto

$$
\begin{gathered}
\frac{\partial \widehat{f}}{\partial t}=c^{\prime}(t) \exp (G)+c(t) \exp (G)\left[-\frac{1}{b^{2}}\left[(x-v t-F t)\left(-v-F-t \frac{\partial F}{\partial t}\right)\right] .\right. \\
v \cdot \nabla_{x} \widehat{f}=v \cdot\left(\frac{\partial \widehat{f}}{\partial x_{1}}, \frac{\partial \widehat{f}}{\partial x_{2}}, \frac{\partial \widehat{f}}{\partial x_{3}}\right) \\
=v_{1} \frac{\partial \widehat{f}}{\partial x_{1}}+v_{2} \frac{\partial \widehat{f}}{\partial x_{2}}+v_{3} \frac{\partial \widehat{f}}{\partial x_{3}}
\end{gathered}
$$

ahora $\frac{\partial \widehat{f}}{\partial x_{1}}=c(t) \exp (G) \frac{\partial}{\partial x_{1}}(G)$ 


$$
\begin{gathered}
\frac{\partial}{\partial x_{1}}(G)=\frac{\partial}{\partial x_{1}}\left\{-\frac{1}{2}\left[\left(\frac{x_{1}-v_{1} t-F_{1} t}{b}\right)^{2}+\left(\frac{x_{2}-v_{2} t-F_{2} t}{b}\right)^{2}+\left(\frac{x_{3}-v_{3} t-F_{3} t}{b}\right)^{2}\right]\right\} \\
=-\frac{1}{b^{2}}\left(x_{1}-v_{1} t-F_{1} t\right)\left(1-t \frac{\partial F_{1}}{\partial x_{1}}\right)
\end{gathered}
$$

entonces

$$
\begin{array}{r}
\frac{\partial \widehat{f}}{\partial x_{1}}=c(t) \exp (G)\left[-\frac{1}{b^{2}}\left(x_{1}-v_{1} t-F_{1} t\right)\left(1-t \frac{\partial F_{1}}{\partial x_{1}}\right)\right], \text { por lo tanto } \\
v \cdot \nabla_{x} \widehat{f}=c(t) \exp (G)\left(-\frac{1}{b^{2}}\right)\left(x_{1}-v_{1} t-F_{1} t\right)\left(1-t \frac{\partial F_{1}}{\partial x_{1}}\right) v_{1} \\
+c(t) \exp (G)\left(-\frac{1}{b^{2}}\right)\left(x_{2}-v_{2} t-F_{2} t\right)\left(1-t \frac{\partial F_{2}}{\partial x_{2}}\right) v_{2} \\
+c(t) \exp (G)\left(-\frac{1}{b^{2}}\right)\left(x_{3}-v_{3} t-F_{3} t\right)\left(1-t \frac{\partial F_{3}}{\partial x_{3}}\right) v_{3} . \\
\mathrm{F} \cdot \nabla_{v} \widehat{f}=F_{1} \frac{\partial \widehat{f}}{\partial v_{1}}+F_{2} \frac{\partial \widehat{f}}{\partial v_{2}}+F_{3} \frac{\partial \widehat{f}}{\partial v_{3}} \\
\frac{\partial \widehat{f}}{\partial v_{1}}=c(t) \exp (G) \frac{\partial}{\partial v_{1}}(G)
\end{array}
$$

ahora

$$
\frac{\partial}{\partial v_{1}}(G)=-\frac{1}{b^{2}}\left(x_{1}-v_{1} t-F_{1} t\right)\left(-t-t \frac{\partial F_{1}}{\partial v_{1}}\right)
$$

entonces

$$
\begin{gathered}
F \cdot \nabla_{v} \widehat{f}=F_{1} c(t) \exp (G)\left(\frac{t}{b^{2}}\right)\left(x_{1}-v_{1} t-F_{1} t\right)\left(1+\frac{\partial F_{1}}{\partial v_{1}}\right) \\
+F_{2} c(t) \exp (G)\left(\frac{t}{b^{2}}\right)\left(x_{2}-v_{2} t-F_{2} t\right)\left(1+\frac{\partial F_{2}}{\partial v_{2}}\right) \\
+F_{3} c(t) \exp (G)\left(\frac{t}{b^{2}}\right)\left(x_{3}-v_{3} t-F_{3} t\right)\left(1+\frac{\partial F_{3}}{\partial v_{3}}\right) .
\end{gathered}
$$




$$
\begin{aligned}
& t \frac{\partial F}{\partial t} \cdot \nabla_{v} \widehat{f}=t\left(\frac{\partial F_{1}}{\partial t}, \frac{\partial F_{2}}{\partial t}, \frac{\partial F_{3}}{\partial t}\right) \cdot\left(\frac{\partial \widehat{f}}{\partial v_{1}}, \frac{\partial \widehat{f}}{\partial v_{2}}, \frac{\partial \widehat{f}}{\partial v_{3}}\right) \\
& =t \frac{\partial F_{1}}{\partial t} \frac{\partial \widehat{f}}{\partial v_{1}}+t \frac{\partial F_{2}}{\partial t} \frac{\partial \widehat{f}}{\partial v_{2}}+t \frac{\partial F_{3}}{\partial t} \frac{\partial \widehat{f}}{\partial v_{3}} \\
& =t \frac{\partial F_{1}}{\partial t}\left[c(t) \exp (G)\left(-\frac{1}{b^{2}}\right)\left(x_{1}-v_{1} t-F_{1} t\right)\left(-t-t \frac{\partial F_{1}}{\partial v_{1}}\right)\right] \\
& +t \frac{\partial F_{2}}{\partial t}\left[c(t) \exp (G)\left(-\frac{1}{b^{2}}\right)\left(x_{2}-v_{2} t-F_{2} t\right)\left(-t-t \frac{\partial F_{2}}{\partial v_{2}}\right)\right] \\
& +t \frac{\partial F_{3}}{\partial t}\left[c(t) \exp (G)\left(-\frac{1}{b^{2}}\right)\left(x_{3}-v_{3} t-F_{3} t\right)\left(-t-t \frac{\partial F_{3}}{\partial v_{3}}\right)\right] .
\end{aligned}
$$

Como queremos que $\widehat{f}$ satisfaga la ecuación (1.1), se debe tener que

$$
\frac{\partial \widehat{f}}{\partial t}+v \cdot \nabla_{x} \widehat{f}+F \cdot \nabla_{v} \widehat{f}+t \frac{\partial F}{\partial t} \cdot \nabla_{v} \widehat{f}=E(\widehat{f})
$$

así teniendo en cuenta (1.2), (1.3) y (1.4) en los calculos anteriores tenemos,

$$
c^{\prime}(t) \exp (G)=E(\widehat{f}) \text {. }
$$

Considerando el operador de Enskog

$$
\begin{aligned}
E(f)(t, x, v)= & a^{2} \int_{\mathbf{R}^{3} \times S_{+}^{2}} Y(f) B(\eta, w-v) f\left(t, x, v^{\prime}\right) f\left(t, x+a \eta, w^{\prime}\right) d \eta d w \\
& -a^{2} f(t, x, v) \int_{\mathbf{R}^{3} \times S_{+}^{2}} Y(f) B(\eta, w-v) f(t, x-a \eta, w) d \eta d w,
\end{aligned}
$$

donde $a$ es el diámetro de la esfera considerada en el modelo de gases de esferas duras, teniendo en cuenta la hipótesis 1. para el funcional $Y$ y la forma como está definida $\widehat{f}$, tenemos

$$
\begin{gathered}
(\widehat{f})(t, x, v)= \\
a^{2} \int_{\mathbf{R}^{3} \times S_{+}^{2}} Y(\widehat{f}) B(\eta, w-v) \widehat{f}\left(t, x, v^{\prime}\right) \widehat{f}\left(t, x+a \eta, w^{\prime}\right) d \eta d w \\
-a^{2} \widehat{f}(t, x, v) \int_{\mathbf{R}^{3} \times S_{+}^{2}} Y(\widehat{f}) B(\eta, w-v) \widehat{f}(t, x-a \eta, w) d \eta d w \\
\leq a^{2} M \int_{\mathbf{R}^{3} \times S_{+}^{2}} B(\eta, w-v) \widehat{f}\left(t, x, v^{\prime}\right) \widehat{f}\left(t, x+a \eta, w^{\prime}\right) d \eta d w \\
-a^{2} \widehat{f}(t, x, v) M \int_{\mathbf{R}^{3} \times S_{+}^{2}} B(\eta, w-v) \widehat{f}(t, x-a \eta, w) d \eta d w \\
\leq a^{2} M \int_{\mathbf{R}^{3} \times S_{+}^{2}} B(\eta, w-v) \widehat{f}\left(t, x, v^{\prime}\right) \widehat{f}\left(t, x+a \eta, w^{\prime}\right) d \eta d w \\
+a^{2} \widehat{f}(t, x, v) M \int_{\mathbf{R}^{3} \times S_{+}^{2}} B(\eta, w-v) \widehat{f}(t, x-a \eta, w) d \eta d w \\
\left.\leq 2 a^{2} M c^{2}(t) \int_{\mathbf{R}^{3} \times S_{+}^{2}} B(\eta, w-v)\right) d \eta d w
\end{gathered}
$$


Por la hipótesis hecha a $B$ existe un $0 \leq L<\infty$ tal que

$$
\int_{\mathbf{R}^{3} \times S_{+}^{2}}|B(\eta, w-v)| d \eta d w \leq L
$$

luego

$$
E(\widehat{f})(t, x, v) \leq 2 a^{2} L M c^{2}(t)
$$

por lo tanto

$$
c^{\prime}(t) \exp (G) \leq 2 a^{2} L M c^{2}(t)
$$

para esta desigualdad diferencial, las soluciones siempre existen y satisfacen $c(t) \leq \frac{c(0)}{1-2 c(0) a^{2} M L T}$ en efecto,

$$
\begin{gathered}
c^{\prime}(t) \exp (G) \leq 2 a^{2} L M c^{2}(t) \\
c^{-2}(t) c^{\prime}(t) \leq \frac{2 a^{2} L M}{\exp (G)} \leq 2 a^{2} L M \\
-\left[c^{-1}(t)\right]^{\prime} \leq 2 a^{2} L M \\
{\left[c^{-1}(t)\right]^{\prime} \geq-2 a^{2} L M}
\end{gathered}
$$

integrando esta última expresión con respecto a $t$

$$
c^{-1}(t)-c^{-1}(0) \geq-2 a^{2} L M t
$$

así

$$
\frac{1}{c(t)} \geq \frac{1}{c(0)}-2 a^{2} L M t
$$

de donde

$$
0 \leq c(t) \leq \frac{c(0)}{1-2 c(0) a^{2} L M t} \leq \frac{c(0)}{1-2 c(0) a^{2} L M T}
$$

con $T \geq t \geq 0$.

Definimos la aplicación

$\Lambda: \quad V \longrightarrow V$

$$
f \longrightarrow \Lambda(f)=\psi
$$

de tal modo que 


$$
\left\{\begin{array}{c}
\partial_{t} \psi+v \cdot \nabla_{x} \psi+\left(F+t \partial_{t} F\right) \cdot \nabla_{v} \psi=E(f) \\
\psi(0, \cdot)=f_{0}
\end{array}\right.
$$

Si $f_{0} \geq 0$, entonces $\psi \geq 0$, esto se demuestra mediante el esquema de Kaniel - Shinbrot (ver [5]).

Si $f \in V$ entonces $\Lambda(f) \in V$, en efecto, como $f \in V$ se tiene que

$$
\begin{aligned}
\partial_{t} \psi+v \cdot \nabla_{x} \psi+\left(F+t \partial_{t} F\right) \cdot \nabla_{v} \psi & =E(f) \\
& \leq E(\widehat{f}) \\
& =\partial_{t} \widehat{f}+v \cdot \nabla_{x} \widehat{f}+\left(F+t \partial_{t} F\right) \cdot \nabla_{v} \widehat{f}
\end{aligned}
$$

entonces $\psi \leq \widehat{f}$, es decir, $\Lambda(f) \in V$ ( aqui usamos la hipótesis de monotonia del operador $Y$ ).

Sean $\psi_{1}=\Lambda(f)$ y $\psi_{2}=\Lambda(g)$, entonces $\left|\psi_{1}-\psi_{2}\right|=|\Lambda(f)-\Lambda(g)|$. Definamos $\psi^{\#}(t, x, v)=\psi(t, x+v t, v+F t)$ luego

$$
\frac{d}{d t} \psi^{\#}(t, x, v)=\partial_{t} \psi+v \cdot \nabla_{x} \psi+\left(F+t \partial_{t} F\right) \cdot \nabla_{v} \psi=E(f)
$$

es decir,

$$
\psi^{\#}(t, x, v)=f(0, x, v)+\int_{0}^{T} E(f) d t
$$

por la definición de $\psi^{\#}$ y (2.1). Esto me indica, que para definir el operador Lipzchitz continuo, necesito que f este definida en $(0, x, v)$ y no el campo $F$, ahora

$$
\begin{aligned}
& \left|\psi_{1}^{\#}(t, x, v)-\psi_{2}^{\#}(t, x, v)\right|=\left|\int_{0}^{T}(E(f)-E(g)) d t\right| \\
& \left\|\psi_{1}^{\#}-\psi_{2}^{\#}\right\|_{V} \leq \frac{1}{\hat{f}(t, x, v)} \int_{0}^{T}|E(f)-E(g)| d t
\end{aligned}
$$

ahora calculamos 


$$
\begin{aligned}
& |E(f)(t, x, v)-E(g)(t, x, v)|= \\
& \begin{array}{l}
=a^{2} \int_{\mathbf{R}^{3} \times S_{+}^{2}} Y(f) B(\eta, w-v) f\left(t, x, v^{\prime}\right) f\left(t, x+a \eta, w^{\prime}\right) d \eta d w \\
-a^{2} f(t, x, v) \int_{\mathbf{R}^{3} \times S^{2}} Y(f) B(\eta, w-v) f(t, x-a \eta, w) d \eta d w
\end{array} \\
& -a^{2} \int_{\mathbf{R}^{3} \times S_{+}^{2}} Y(g) B(\eta, w-v) g\left(t, x, v^{\prime}\right) g\left(t, x+a \eta, w^{\prime}\right) d \eta d w \\
& +a^{2} g(t, x, v) \int_{\mathbf{R}^{3} \times S_{+}^{2}} Y(g) B(\eta, w-v) g(t, x-a \eta, w) d \eta d w \\
& =\mid a^{2} \int_{\mathbf{R}^{3} \times S_{+}^{2}} B(\eta, w-v)\left[Y(f) f\left(t, x, v^{\prime}\right) f\left(t, x+a \eta, w^{\prime}\right)\right. \\
& -Y(f) f\left(t, x, v^{\prime}\right) g\left(t, x+a \eta, w^{\prime}\right)+Y(f) f\left(t, x, v^{\prime}\right) g\left(t, x+a \eta, w^{\prime}\right) \\
& -Y(f) g\left(t, x, v^{\prime}\right) g\left(t, x+a \eta, w^{\prime}\right) \\
& \left.+[Y(f)-Y(g)] g\left(t, x, v^{\prime}\right) g\left(t, x+a \eta, w^{\prime}\right)\right] d \eta d w \\
& +a^{2}[g(t, x, v)-f(t, x, v)] \int_{\mathbf{R}^{3} \times S_{+}^{2}} Y(g) B(\eta, w-v) g(t, x-a \eta, w) d \eta d w \\
& +a^{2} f(t, x, v)\left[\int_{\mathbf{R}^{3} \times S_{+}^{2}} Y(g) B(\eta, w-v) g(t, x-a \eta, w) d \eta d w\right. \\
& +\int_{\mathbf{R}^{3} \times S_{+}^{2}} Y(g) B(\eta, w-v) f(t, x-a \eta, w) d \eta d w \\
& -\int_{\mathbf{R}^{3} \times S_{+}^{2}} Y(g) B(\eta, w-v) f(t, x-a \eta, w) d \eta d w \\
& \left.-\int_{\mathbf{R}^{3} \times S_{+}^{2}} Y(f) B(\eta, w-v) f(t, x-a \eta, w) d \eta d w\right]
\end{aligned}
$$

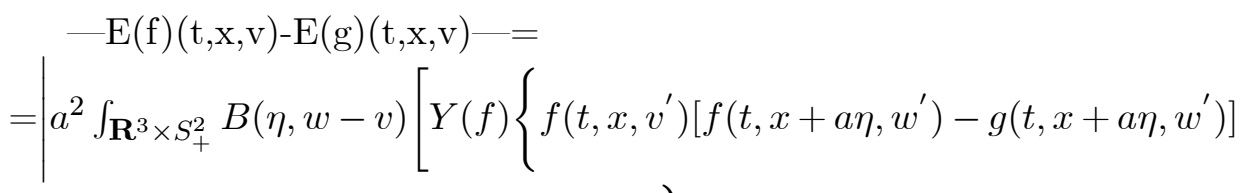

$$
\begin{aligned}
& \left.+\left[f\left(t, x, v^{\prime}\right)-g\left(t, x, v^{\prime}\right)\right] g\left(t, x+a \eta, w^{\prime}\right)\right\} \\
& \left.+[Y(f)-Y(g)] g\left(t, x, v^{\prime}\right) g\left(t, x+a \eta, w^{\prime}\right)\right] d \eta d w \\
& +a^{2}[g(t, x, v)-f(t, x, v)] \int_{\mathbf{R}^{3} \times S_{+}^{2}} Y(g) B(\eta, w-v) g(t, x-a \eta, w) d \eta d w \\
& +a^{2} f(t, x, v) \int_{\mathbf{R}^{3} \times S_{+}^{2}} Y(g) B(\eta, w-v)[g(t, x-a \eta, w)-f(t, x-a \eta, w)] d \eta d w \\
& -a^{2} f(t, x, v) \int_{\mathbf{R}^{3} \times S_{+}^{2}}[Y(g)-Y(f)] B(\eta, w-v) f(t, x-a \eta, w) d \eta d w
\end{aligned}
$$


como $f, g \in V$ y además por la hipótesis 1. impuesta al funcional $Y$

$$
\begin{aligned}
& \quad-\mathrm{E}(\mathrm{f})(\mathrm{t}, \mathrm{x}, \mathrm{v})-\mathrm{E}(\mathrm{g})(\mathrm{t}, \mathrm{x}, \mathrm{v})-\leq \\
& a^{2} \int_{\mathbf{R}^{3} \times S_{+}^{2}} B(\eta, w-v)\left[M \left\{\widehat{f}\left(t, x, v^{\prime}\right)\left|f\left(t, x+a \eta, w^{\prime}\right)-g\left(t, x+a \eta, w^{\prime}\right)\right| d \eta d w\right.\right. \\
& \left.+\left|f\left(t, x, v^{\prime}\right)-g\left(t, x, v^{\prime}\right)\right| \widehat{f}\left(t, x+a \eta, w^{\prime}\right)\right\} \\
& \left.+|Y(f)-Y(g)| \widehat{f}\left(t, x, v^{\prime}\right) \widehat{f}\left(t, x+a \eta, w^{\prime}\right)\right] d \eta d w \\
& +a^{2}|g(t, x, v)-f(t, x, v)| \int_{\mathbf{R}^{3} \times S_{+}^{2}} M B(\eta, w-v) \widehat{f}(t, x-a \eta, w) d \eta d w \\
& +a^{2} \widehat{f}(t, x, v) \int_{\mathbf{R}^{3} \times S_{+}^{2}} M B(\eta, w-v)[g(t, x-a \eta, w)-f(t, x-a \eta, w)] d \eta d w \\
& -a^{2} \widehat{f}(t, x, v) \int_{\mathbf{R}^{3} \times S_{+}^{2}}|Y(g)-Y(f)| B(\eta, w-v) \widehat{f}(t, x-a \eta, w) d \eta d w \mid
\end{aligned}
$$

teniendo en cuenta la definición de la norma en el espacio $V$

$$
\begin{aligned}
& -\mathrm{E}(\mathrm{f})(\mathrm{t}, \mathrm{x}, \mathrm{v})-\mathrm{E}(\mathrm{g})(\mathrm{t}, \mathrm{x}, \mathrm{v})-\leq \\
& a^{2} \int_{\mathbf{R}^{3} \times S_{+}^{2}} B(\eta, w-v)\left[M \left\{\widehat{f}\left(t, x, v^{\prime}\right) \widehat{f}\left(t, x+a \eta, w^{\prime}\right)\|f-g\|_{V}\right.\right. \\
& \left.+\widehat{f}\left(t, x, v^{\prime}\right) \widehat{f}\left(t, x+a \eta, w^{\prime}\right)\|f-g\|_{V}\right\} \\
& \left.+|Y(f)-Y(g)| \widehat{f}\left(t, x, v^{\prime}\right) \widehat{f}\left(t, x+a \eta, w^{\prime}\right)\right] d \eta d w \\
& +a^{2} \widehat{f}(t, x, v)\|f-g\|_{V} \int_{\mathbf{R}^{3} \times S_{+}^{2}} M B(\eta, w-v) \widehat{f}(t, x-a \eta, w) d \eta d w \\
& +a^{2} \widehat{f}(t, x, v) \int_{\mathbf{R}^{3} \times S_{+}^{2}} M B(\eta, w-v) \widehat{f}(t, x-a \eta, w)\|f-g\|_{V} d \eta d w \\
& -a^{2} \widehat{f}(t, x, v) \int_{\mathbf{R}^{3} \times S_{+}^{2}}|Y(g)-Y(f)| B(\eta, w-v) \widehat{f}(t, x-a \eta, w) d \eta d w \mid
\end{aligned}
$$

entonces

$$
\begin{aligned}
& -\mathrm{E}(\mathrm{f})(\mathrm{t}, \mathrm{x}, \mathrm{v})-\mathrm{E}(\mathrm{g})(\mathrm{t}, \mathrm{x}, \mathrm{v})-\leq \\
& \mid 2 a^{2} M\|f-g\|_{V}\left[\int_{\mathbf{R}^{3} \times S_{+}^{2}} B(\eta, w-v)\left(\widehat{f}\left(t, x, v^{\prime}\right) \widehat{f}\left(t, x+a \eta, w^{\prime}\right)\right) d \eta d w\right.
\end{aligned}
$$




$$
\begin{aligned}
& \left.+\int_{\mathbf{R}^{3} \times S_{+}^{2}} B(\eta, w-v)(\widehat{f}(t, x, v) \widehat{f}(t, x-a \eta, w)) d \eta d w\right] \\
& +\mathrm{a}^{2} K\|f-g\|_{V}\left[\int_{\mathbf{R}^{3} \times S_{+}^{2}} B(\eta, w-v)\left(\widehat{f}\left(t, x, v^{\prime}\right) \widehat{f}\left(t, x+a \eta, w^{\prime}\right)\right) d \eta d w\right. \\
& \left.-\int_{\mathbf{R}^{3} \times S_{+}^{2}} B(\eta, w-v)(\widehat{f}(t, x, v) \widehat{f}(t, x-a \eta, w)) d \eta d w\right] \mid
\end{aligned}
$$

donde se ha utilizado la hipótesis 3. del funcional $Y$, por lo tanto

$$
\begin{aligned}
& \left\|\psi_{1}^{\#}-\psi_{2}^{\#}\right\|_{V} \leq \\
& \int_{0}^{T} \mid a^{2}(2 M+K)\|f-g\|_{V} \int_{\mathbf{R}^{3} \times S_{+}^{2}} B(\eta, w-v) \frac{\widehat{f}\left(t, x, v^{\prime}\right) \widehat{f}\left(t, x+a \eta, w^{\prime}\right)}{\widehat{f}(t, x, v)} d \eta d w \\
& +a^{2}(2 M-K)\|f-g\|_{V} \int_{\mathbf{R}^{3} \times S_{+}^{2}} B(\eta, w-v) \frac{\widehat{f}(t, x, v) \widehat{f}(t, x-a \eta, w)}{\widehat{f}(t, x, v)} d \eta d w \mid d t
\end{aligned}
$$

entonces

$$
\begin{aligned}
& \left\|\psi_{1}^{\#}-\psi_{2}^{\#}\right\|_{V} \leq \\
& a^{2}(2 M+K)\|f-g\|_{V} \int_{0}^{T} \int_{\mathbf{R}^{3} \times S_{+}^{2}}\left|B(\eta, w-v)\|\widehat{f}\| \widehat{f}\left(t, x+a \eta, w^{\prime}\right)\right| d \eta d w d t \\
& +a^{2}(2 M-K)\|f-g\|_{V} \int_{0}^{T} \int_{\mathbf{R}^{3} \times S_{+}^{2}}|B(\eta, w-v)\|\widehat{f}\| \widehat{f}(t, x-a \eta, w)| d \eta d w d t \\
& \left\|\psi_{1}^{\#}-\psi_{2}^{\#}\right\|_{V} \leq \\
& a^{2}(2 M+K)\|f-g\|_{V} \int_{0}^{T} \int_{\mathbf{R}^{3} \times S_{+}^{2}}\left|B(\eta, w-v) \widehat{f}\left(t, x+a \eta, w^{\prime}\right)\right| d \eta d w d t \\
& +a^{2}(2 M-K)\|f-g\|_{V} \int_{0}^{T} \int_{\mathbf{R}^{3} \times S_{+}^{2}}|B(\eta, w-v) \widehat{f}(t, x-a \eta, w)| d \eta d w d t \\
& \leq a^{2}(2 M+K) C(t)\|f-g\|_{V} L+a^{2}(2 M-K) c(t)\|f-g\|_{V} L \\
& =4 a^{2} c(t) L M\|f-g\|_{V}
\end{aligned}
$$

y así

$$
\left\|\psi_{1}^{\#}-\psi_{2}^{\#}\right\|_{V} \leq 4 M a^{2} L c(t)\|f-g\|_{V}
$$


Ahora $4 M L a^{2} c(t)<4 M L a^{2}\left[\frac{c(0)}{1-2 c(0) a^{2} M L T}\right] \leq 1$, esto es $a<\sqrt{\frac{1}{2 M L c(0)(2+T)}}$, por tanto por el teorema del punto fijo de Banach tenemos que ,existe un único $f \in V$ tal que $f(t, x, v)=f(0, x, v)+\int_{0}^{T} E(f) d t$, es decir, f es solución de la ecuación con condicion inicial $f(0, x, v)=f_{0}(x, v)$.

Agradecemos al referee por sus multiples sugerencias para mejorar el artículo.

\section{Bibliografía}

[1] Amador Xavier El modelo de Friedman-Robertson-Walker, http://exactphisicist.tripod.com/Bigbang.html.

[2] Asano Kiyoshi On the global solution of the initial-boundary value problems for the Boltzmann equation with external force, T. T. S. P., pp. 735-761, (1987).

[3] Asano Kiyoshi Local solutions to initial and initial boundary value problem for the Boltzmann Equation with and external force,J. Math. Kyoto Univ., vol 24, pp. 225-238, (1984) .

[4] Bellomo N, Lachowicz M, Palczwiski and Toscani G. On the initial value problem for the Boltzmann equation with force term, T.T.S.P.,vol 18, pp. 87-102, (1989).

[5] Bellomo N,Lectures notes on the Mathematical theory of the Boltzmann Equation, World Scientific S. A. M. A. S., vol 33.

[6] Galeano R., Orozco B., Vasquez O.Solución Distribucional de la Ecuación de Enskog para un dato cerca al Maxweliano, Revista Digital Matemtica Educación e Internet, Vol. 8, N 1, (2007).

[7] Galeano R., Perez H.Problema de Cauchy para la ecuación de Enskog no lineal con término fuerza, Foro-Red-Mat, UNAM.,vol 13, (2003).

[8] Grunfeld C.On the nonlinear Boltzmann equation with force term, T. T. S. P., vol 14, pp. 291-322, (1985).

[9] Hamdache K.These, univ. Pierre et Marie Curie, Paris, (1988). 
[10] Lachowicz M.Sul problema al valore iniziale per l'equazione di Enskog con campo externo, Bol. Un. Mat. Ital., (2000).

[11] Mischler S. and Perthame B.Boltzmann Equation with Infinite Energy: Renormalized Solutions and Distributional Solutions for small initial data and initial data close to a Maxwellian, SIAM J. Math. Anal., vol 28, pp. 1015-1027, (2000).

[12] Zhang Xianwen.The spectrum of the linear Boltzmann operator with an external field, T. T. S. P., pp. 699-710, (2000).

\section{Rafael Galeano Andrades}

Instituto de Matemáticas Aplicadas

Universidad de Cartagena

Cartagena,

Colombia

e-mail : rgaleanoa@unicartagena.edu.co

\section{Bernardo Orozco Herrera}

Instituto de Matemáticas Aplicadas, Universidad de Cartagena

Cartagena,

Colombia

e-mail : borozcoh@unicartagena.edu.co

and

María Ofelia Vásquez Avila

Instituto de Matemáticas Aplicadas, Universidad de Cartagena,

Cartagena,

Colombia

e-mail : mvasqueza@unicartagena.edu.co 